\title{
Using Near-Infrared Spectroscopy for the noninvasive identification of five pharmaceutical active substances in sealed vials
}

\author{
M. Ulmschneider ${ }^{1, *}$, A. Wunenburger ${ }^{1}$ and E. Pénigault ${ }^{2}$ \\ ${ }^{1}$ Pharmaceutical Quality Control and Assurance, F. Hoffmann - La Roche Ltd, CH-4070 Basle, Switzerland \\ ${ }^{2}$ Laboratoire de Photochimie Générale, UMR 7525 du CNRS, ENSCMu, 3 rue Alfred Werner, \\ F-68093 Mulhouse Cedex, France
}

\begin{abstract}
In this study, a method combining near-infrared spectroscopy and the corresponding chemometric model was developed for the noninvasive identification of five pharmaceutical active substances in sealed vials: ceftriaxone disodium salt sterile, saquinavir mesylate, saquinavir base, nelfinavir mesylate and neuraminidase inhibitor. This method is very specific, fast and nondestructive. It is an alternative to the identification performed hitherto by infrared spectroscopy. Almost no sample preparation or cleaning operation was required apart from the filling of the actives in vials. Measurements were performed on a horizontal sample desk with a monochromator NIR spectrometer.
\end{abstract}

Keywords. NIR spectroscopy - pharmaceutical active substances - noninvasive identification - monochromator.

\section{Introduction}

Near-Infrared (NIR) spectroscopy is gaining increasing interest as an alternative analytical method in the pharmaceutical industry $[1,2,3]$. For instance, rapid identification of raw materials is a classical application for NIR spectroscopy $[4,5,6]$. The fundamental advantages of measurements in the near infrared spectral region make NIR an obvious choice for quality control applications in a production environment [7,8]. Since NIR-spectra contain both chemical and physical information, simple and direct interpretation is not possible. Appropriate computerized analytical methods are necessary to solve the problem of selectivity in mathematical terms and unscramble concealed and interfering spectral relationships.

Overtone and combination vibrations of the mid-infrared range mainly occur in the NIR range (approximately 1000 to $2500 \mathrm{~nm}$ ). As a rule, the intensity of these - generally broad - overlapping bands is weaker by a factor of ca 10 to 100 than the intensity of the fundamental band. This apparent drawback of the NIR range is offset by the fact that larger layer thicknesses can be measured than in the mid-IR range. This means that averaging can occur over a greater sample volume, thus entailing less exacting homogeneity requirements of the sample. Handling of the samples is also simplified. Since the ratio of reflected to absorbed light is high in the NIR range, the technique is particularly suited to measurements of diffuse reflection. In addition, since white glass is not an obstacle to NIR radiation, identity testing can be performed directly through sealed vials. After measurement the samples are still available and no chemicals are used for sample preparation.

According to the current GMP requirements for the pharmaceutical industry, an identity testing of active substances used in selected Roche drugs was decided. The present study is part of that project.

\section{Experimental}

The five pharmaceutical active substances selected at the site of Roche at Basel: ceftriaxone disodium salt sterile, saquinavir mesylate, saquinavir base, nelfinavir mesylate, and neuraminidase inhibitor, were used as pure powdered forms and expected to be homogeneous. They were filled into standard $15 \mathrm{ml}$ white septum vials.

The method retained for computing the NIR model for identity testing relied on an independent cross validation approach. This means that samples were kept out of the calibration stage and only used for prediction during the separate validation stage [9]. The remaining samples were included into the validation set. The latter was independent of the calibration set and used directly for assessing the effectiveness of the calibration. The following table shows the five substances with the corresponding number of samples and the distribution of the samples into calibration set and validation set.

The number of samples was adapted to the current availability of the material to be identified. The substances were

* Correspondence and reprints.

Received June 16, 1999; revised December 14, 1999; accepted December 17, 1999. 


\section{Original articles}

Table I.

\begin{tabular}{lccc}
\hline Roche Substance & Total samples & In calibration set & In validation set \\
\hline Ceftriaxone disodium salt & 132 & 28 & 104 \\
Saquinavir mesylate & 73 & 14 & 59 \\
Saquinavir base & 5 & 2 & 3 \\
Nelfinavir mesylate & 15 & 8 & 7 \\
Neuraminidase inhibitor & 15 & 8 & 7 \\
\hline Total & 240 & 60 & 180 \\
\hline
\end{tabular}

all produced by Roche sites according to strict requirements and control of the production parameters. Variations in quality were excluded for these materials, which complied fully with the specifications.

NIR-spectra were recorded at $4 \mathrm{~nm}$ resolution over the full NIR range from 1100 to $2500 \mathrm{~nm}$ using a NIRSystems 5000 spectrometer equipped with the Rapid Content Sampler supplied by FOSS/NIRSystems which was housed in a laboratory of the Roche Basle Department for Pharmaceutical Quality Control. The spectrometer was a dispersive scanning NIR instrument of the grating type. The measurements were made using the horizontal sample desk in the diffuse reflectance mode. Optics included a tungsten-halogen source lamp, a single monochromator with a holographic diffraction grating, and 6 uncooled lead sulfide detectors. These were distributed circularly at the base of a glass window fitted with an iris for centring. Each sample was placed on this window in the horizontal sample desk and centred over the light beam. Three replicate spectra were recorded for each sample. A complete spectrum was the mean of 32 full range scans requiring ca $40 \mathrm{~s}$ to take into account instrument variations. The apparent density of the samples was varied between measurements by shaking the sample vial. To illustrate the spectral differences between the five substances, example spectra were recorded and plotted in figure 1 . The NIR-spectra were improved by performing a mathematical pretreatment on the data. The second derivative of the spectra was used for model calculations to reduce baseline shifts and improve peak shape and resolution.

\section{Calibration and results}

The spectra from all samples from the calibration set were compiled to build up a spectral library for identification. Each substance was defined as a unique product in the library. The calibration was computed with the qualitative modeling part of the commercially available NSAS/IQ ${ }^{2}$ Software from FOSS/NIRSytems. Each product was considered as a cloud of related spectra within a corresponding extension in absorbance for each recorded wavelength between 1100 and $2500 \mathrm{~nm}$. Computing a spectral matching library yielded a simplified and interpretable description of the main information contained in the spectral database of all collected spectra that formed the library. For that purpose the library programme in NSAS/IQ ${ }^{2}$ calculated the mean spectrum and the standard deviation for each product defined in the original spectral database.

To identify unknown samples with the spectral matching library, another algorithm in NSAS/IQ ${ }^{2}$ estimated a correlation coefficient as a measurement of spectral similarity. Identification by correlation is very sensitive to the position of the bands, so that organic chemical substances with different NIR spectra may easily be identified. The program went back to the individual spectra of the unknown samples and computed the best correlation with a library entry or product. If the best match was above a given threshold, identity was confirmed and recorded. In a first attempt at setting up a spectral matching library, the matching threshold value was set to its default value $(0.85)$ for identification by correlation. This minimum value was helpful to offer a possible structure of the matching library. In a second endeavour this value was again examined and adjusted according to the results and corresponding correlation values of identity tests performed on routine equivalent unknown samples.

With a view to identifying ceftriaxone disodium salt sterile, saquinavir mesylate, saquinavir base, nelfinavir mesylate and neuraminidase inhibitor, the spectral matching library was calculated by using a set of 180 NIR-spectra contained in the database for calibration. Identification of unknown samples was performed by correlation with a threshold value set to $0.95 \%$. A successful identification carried out by spectral matching performs the complete separation of the spectra associated with the different active substances in the original spectral database. This implies non-conflicting product pairs in the calculated spectral matching database. Another major criterion qualifying the result of the calibration is the prediction of the substance associated with the 180 samples of the independent validation set. All the spectra of these samples were correctly identified.

\section{Conclusion}

The methodology described in this paper for the identification of five pharmaceutical active substances can be applied to any kind of powdered organic chemicals, on the assumption that information on the products (concerning the quality and the variability of physical characteristics) is 


\section{Original articles}

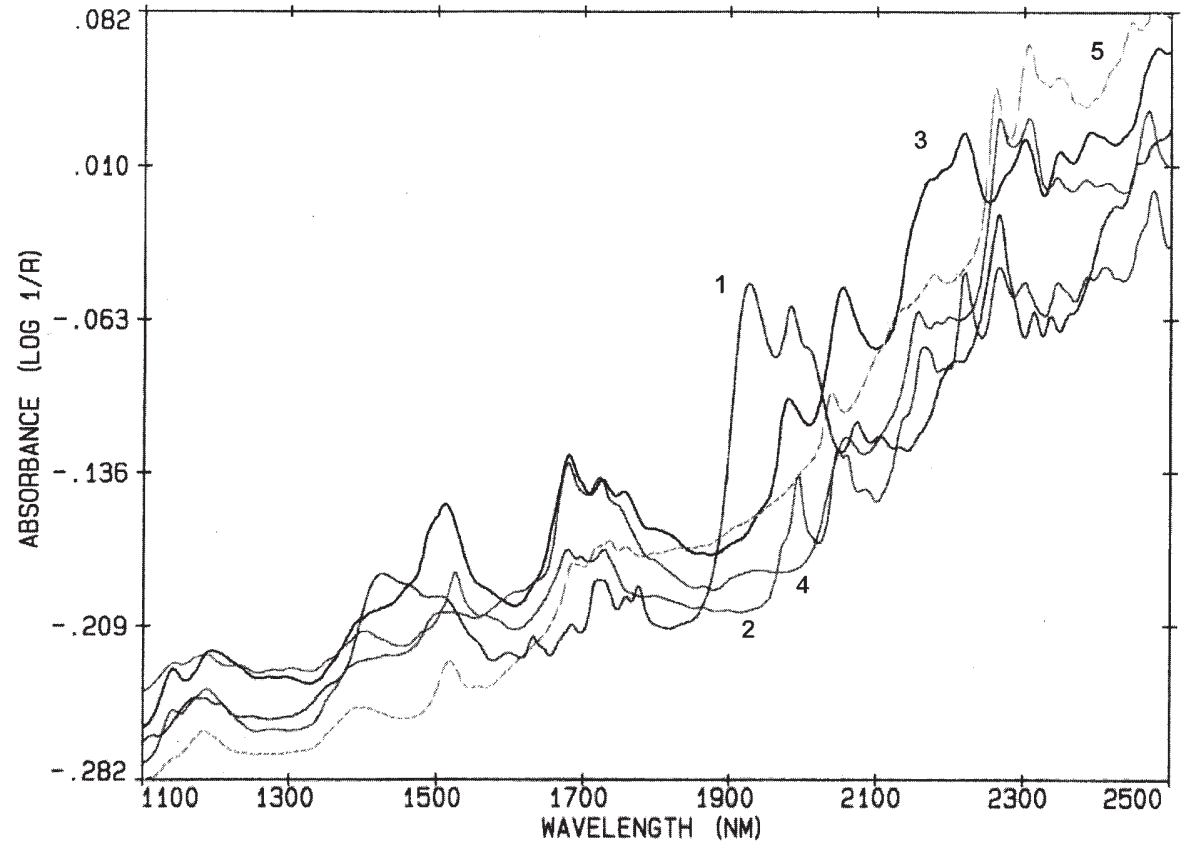

Fig. 1. Differences between the five active substances, ceftriaxone disodium salt sterile (1), saquinavir mesylate (2), saquinavir base (3), nelfinavir mesylate (4) and neuraminidase inhibitor (5), as examplified by their spectra. available. For a given quality and for substances which feature different NIR spectra the number of samples can be kept small. No particular difficulties arise from the transfer of the calibration method described above to a NIR spectrometer of the same make and type. The combination of a calibration stage followed by an independent validation stage is more important to develop NIR models with a good discriminating power. During data analysis a multivariate model describing the available data as well as possible was fitted. Once calibrated, the variations in the data set can be expressed as the sum of a modeled part and a residual part. Any variation observed in the model only results from the actual variations observed in the variables measured during calibration. Any classification tool can, then, be used to predict identity membership.

\section{References}

1. Burns, D. A.; Ciurczak, E. W. Ed. Handbook of Near-Infrared Analysis; Dekker: New York, 1992, Chapter 20.

2. Corti, P.; Savini, L.; Dreassi, E.; Ceramelli, G.; Montecchi, L.; Lonardi, S. Pharmaceutical Acta Helvetica, 1992, 67, 131-142.

3. Plugge, W.; van der Vlies, C. J. of Pharm. \& Biomed. Anal. 1992, 10, 797-803.

4. MacDonald, B.F.; Prebble, K.A. J. of Pharm. \& Biomed. Anal. 1993, 11, 1077-1085.

5. Ulmschneider, M.; Barth, G.; Reder, B. Pharm. Ind., in press.

6. Ulmschneider, M.; Barth, G.; Trenka, E. Pharm. Ind., in press.

7. Knecht, J. Mikrochim. Akta (Suppl.) 1997, 14, 769-771.

8. González, F.; Pous, R. J. of Pharm. \& Biomed. Anal. 1995, 13, 419-423.

9. Ulmschneider, M. In Handbuch der Validierung, Stavros Kronidas, Ed.: Wiley Verlag Chemie: Weinheim, in press. 